\title{
Compatible Poisson Brackets, Quadratic Poisson Algebras and Classical $r$-Matrices
}

\author{
V. Roubtsov and T. Skrypnyk
}

\begin{abstract}
We show that for a general quadratic Poisson bracket it is possible to define a lot of associated linear Poisson brackets: linearizations of the initial bracket in the neighborhood of special points. We prove that the constructed linear Poisson brackets are always compatible with the initial quadratic Poisson bracket.

We apply the obtained results to the cases of the standard quadratic $r$-matrix bracket and to classical "twisted reflection algebra" brackets. In the first case we obtain that there exists only one non-equivalent linearization: the standard linear $r$-matrix bracket and recover well-known result that the standard quadratic and linear $r$-matrix brackets are compatible. We show that there are a lot of non-equivalent linearizations of the classical twisted Reflection Equation Algebra bracket and all of them are compatible with the initial quadratic bracket.
\end{abstract}

\section{Introduction}

The theory of compatible Poisson brackets (or so-called bihamiltonian theory) has appeared almost 30 years ago [1]. Magri had observed the following highly nontrivial fact: a linear combination of two Poisson brackets (or two Poisson tensors) is not always again a Poisson but demands an additional compatibility condition (the annihilation of mutual Schouten brackets for two Poisson tensors). The theory of such compatible brackets (or in other terminology of linear Poisson pencils) was developed later in many papers (see for example [2-6]). Compatibility conditions of Lie-Poisson brackets on finite-dimensional spaces was recently systematically studied in [7] in the assumption that one of the brackets is semisimple.

In the present paper we study linear Poisson pencils of quadratic and linear Poisson brackets. Although there exists a lot of examples of such compatible brackets in literature (see for example [4, 14-16]), there is no (up to our knowledge) a general compatibility theory for such the brackets .

\footnotetext{
T. Skrypnyk (凶)

International School for Advanced Studies, via Beirut 2-4, 34014 Trieste, Italy and Bogolyubov Institute for Theoretical Physics, Metrolohichna str.14-b, Kiev 03680, Ukraine

e-mail: skrypnyk@sissa.it

B. Kruglikov et al. (eds.), Differential Equations: Geometry, Symmetries and Integrability: 
The central idea in this paper is the idea of a linearization which comes from the Poisson-Lie group theory. In more detail, we show that it is possible to construct compatible linear and quadratic Poisson brackets by a linearization of the quadratic bracket in the neighborhood of zeros of their right-hand sides. For each of such zero it is possible to define a linear Poisson bracket which, as we will show, is automatically compatible with the initial quadratic one. We consider the application of this result to the theory of integrable systems in a spirit of the Lenard-Magri scheme [1]. In particular, we obtain a set of commuting functions with respect to both linear and quadratic brackets (we call them "integrals" or "hamiltonians") starting from polynomial Casimirs. It is necessary to emphasize that different choice of zeros of the quadratic Poisson structure may produce non-equivalent linearizations and nonequivalent sets of commuting hamiltonians. We show that the hamiltonian dynamics with respect to one of these hamiltonians of degree $k$ and quadratic bracket can be re-written in the terms of hamiltonian dynamics with respect to a hamiltonian of the degree $k+1$ and the corresponding linear bracket. The last statement has a conceptual importance from the point of view of classical dynamical systems: it means that on the classical level the quadratic Poisson bracket produce the same integrable dynamics as its linearization.

We apply the obtained results to a case of the standard quadratic $r$-matrix bracket and after that to the classical "twisted Reflection Equation Algebra" brackets. We consider spectral parameter-dependent case, though the same results evidently holds true for the spectral parameter-independent situation. The geometry of compatible linear and quadratic brackets associated with a constant $r$-matrix was considered in [8]. The case of constant Reflection Equation Algebra bracket was extensively studied in [9].

In the case of the standard quadratic $r$-matrix bracket $[10,11]$ (in the present paper for the sake of simplicity we consider only the case of $g l(n)$-valued $T$-matrices):

$$
\left\{T_{1}(\lambda), T_{2}(\mu)\right\}_{2}=\left[r_{12}(\lambda-\mu), T_{1}(\lambda) T_{2}(\mu)\right],
$$

we show that its linearizations in the neighborhood of its different zeros produce the standard linear $r$-matrix bracket $[10,11]$ :

$$
\left\{T_{1}(\lambda), T_{2}(\mu)\right\}_{1}=\left[r_{12}(\lambda-\mu), T_{1}(\lambda)+T_{2}(\mu)\right]
$$

or isomorphic to it brackets. In this way we obtain that brackets (1) and (2) are compatible for the arbitrary choice of the $r$-matrix $r(\lambda-\mu)$ satisfying classical Yang-Baxter equation $[10,12,13]$ and for the arbitrary choice of the "monodromy" matrices $T(\lambda)$ satisfying these brackets. The compatibility holds true both for special matrices $T(\lambda)$ that define structure of the finite dimensional quadratic algebra (e.g. those with simple poles in some fixed set of points) and for general meromorphic $T(\lambda)$ that define a structure of a infinite dimensional quadratic algebra. This result generalizes a set of recent results [14-16] about the compatibility of the linear and quadratic $r$-matrix brackets for the cases of the Belavins classical elliptic $r$-matrix [17] and monodromy matrices $T(\lambda)$ possessing simple poles. In the 
present paper we show that $d(\lambda)=\operatorname{det} T(\lambda)$ is a generating function of the Casimir functions of the brackets (1) for any classical $r$-matrix $r(\lambda-\mu)$ taking the values in $\operatorname{sl}(n) \otimes s l(n)$. This fact is certainly known but its rigorous proof seems to be absent in literature. We produce generating functions of commutative integrals of the both of the above brackets applying argument-shift method to the function $d(\lambda)$. We show that generating functions of integrals of order $k$ produce the same integrable dynamics with respect to the quadratic brackets as generating functions of order $k+1$ with respect to the linear bracket.

The main class of our examples is connected with the classical twisted reflection algebra:

$$
\begin{aligned}
\left\{\mathcal{T}_{1}(\lambda), \mathcal{T}_{2}(\mu)\right\}_{2}= & r_{12}(\lambda-\mu) \mathcal{T}_{1}(\lambda) \mathcal{T}_{2}(\mu)-\mathcal{T}_{1}(\lambda) r_{12}^{\sigma_{1}}(-\lambda-\mu) \mathcal{T}_{2}(\mu)- \\
& -\mathcal{T}_{2}(\mu) r_{12}^{\sigma_{2}}(\lambda+\mu) \mathcal{T}_{1}(\lambda)+\mathcal{T}_{1}(\lambda) \mathcal{T}_{2}(\mu) r_{12}^{\sigma_{1} \sigma_{2}}(\mu-\lambda)
\end{aligned}
$$

where $\sigma$ is a second order automorphism of $g l(n)$. (In in the case $\sigma=1$ brackets (3) coincide with the classical limits of standard Reflection Equation algebras $[18,19]$.) We show that there are non-equivalent "linearizations" of these brackets determined by the non-dynamical matrices $K(\lambda)$, where $K(\lambda)$ satisfies the following equation:

$$
\begin{array}{r}
r_{12}(\lambda-\mu) K_{1}(\lambda) K_{2}(\mu)-K_{1}(\lambda) r_{12}^{\sigma_{1}}(-\lambda-\mu) K_{2}(\mu)= \\
=K_{2}(\mu) r_{12}^{\sigma_{2}}(\lambda+\mu) K_{1}(\lambda)-K_{1}(\lambda) K_{2}(\mu) r_{12}^{\sigma_{1} \sigma_{2}}(\mu-\lambda) .
\end{array}
$$

These linear brackets have in terms of new variables $\mathcal{T}^{K}(\lambda) \equiv \mathcal{T}(\lambda) K^{-1}(\lambda)$ the following explicit form:

$$
\begin{aligned}
\left\{\mathcal{T}_{1}^{K}(\lambda), \mathcal{T}_{2}^{K}(\mu)\right\}_{1}= & {\left[r_{12}(\lambda-\mu)-K_{2}(\mu) r_{12}^{\sigma_{2}}(\lambda+\mu) K_{2}^{-1}(\mu), \mathcal{T}_{1}^{K}(\lambda)\right]-} \\
& -\left[r_{21}(\mu-\lambda)-K_{1}(\lambda) r_{21}^{\sigma_{1}}(\lambda+\mu) K_{1}^{-1}(\lambda), \mathcal{T}_{2}^{K}(\mu)\right] .
\end{aligned}
$$

All linearizations corresponding to different $K(\lambda)$ are compatible with the initial twisted reflection algebra bracket (3). In the case of $\mathfrak{g}=s l(2)$, elliptic $r$-matrix $r(\lambda-\mu)$ of Sklyanin [10], trivial automorphism $\sigma$ and $K(\lambda)=1$ such compatibility was observed in the first time in [15]. In the general case our result seems to be new.

Let us also note that the bracket (5) is an example of the linear Poisson bracket governed by the non-skew symmetric $r$-matrices $r(\lambda, \mu)$ [20-22]. In our case we have:

$$
r(\lambda, \mu)=r^{\sigma, K}(\lambda, \mu) \equiv r_{12}(\lambda-\mu)-K_{2}(\mu) r_{12}^{\sigma_{2}}(\lambda+\mu) K_{2}^{-1}(\mu)
$$

The classical $r$-matrix $r^{\sigma, K}(\lambda, \mu)$ may be obtained also from some other considerations [23].

Let us emphasize that each solution of (4) produces its own set of commuting with respect to the bracket (3) functions. This gives one possibility to obtain a lot of different commuting families of functions with respect to the same bracket (3). We 
consider in detail the families of solutions of (4) and the families of the corresponding linear structures given by the $r$-matrix (6) in the simplest case of $\mathfrak{g}=\operatorname{sl}(2)$ and elliptic $r$-matrix of Sklyanin [10]. We also show that $D(\lambda)=\operatorname{det} \mathcal{T}(\lambda)$ is a generating function of the Casimir functions of the brackets (3) for any classical $r$-matrix $r(\lambda-\mu)$. We produce generating functions of commutative integrals of the above brackets applying argument-shift method to the function $D(\lambda)$. We show that generating functions of the integrals of order $k$ produce the same integrable dynamics with respect to the quadratic bracket (3) as generating functions of order $k+1$ with respect to the linear bracket (5).

The structure of the paper is the following: in Sect. 2 we consider general theory of consistent quadratic and linear Poisson brackets. In Sect. 3 we apply the results of Sect. 2 to the quadratic brackets (1) and (3) investigate their linearizations and corresponding algebras of integrals.

\section{Compatible Brackets and Linearization: General Case}

Let $M$ be a (finite or infinite-)dimensional manifold. Let $\left\{T_{i} \mid i \in I\right\}$ (where $I$ is some finite set in the finite-dimensional case, or $\mathbf{Z}$ in the infinite-dimensional case) be the coordinate functions in some local chart on this manifold. Let us consider a quadratic Poisson bracket on $M$, admitting in these coordinates the following explicit form:

$$
\left\{T_{i}, T_{j}\right\}_{2}=\sum_{k, l \in I} C_{i j}^{k l} T_{k} T_{l} .
$$

Here the tensor $C_{i j}^{k l}$ is assumed to be such that the sum (in infinite-dimensional case) is correctly defined. In this paper we will not study the global aspects of the manifold $M$ geometry, preferring to work in the fixed local chart.

We will now linearize this quadratic bracket. In other words we will obtain a linear Poisson bracket which is in a natural way associated with the bracket (7) and is defined on the tangent space $V$ in some fixed point $c \in M$ with the coordinates $c_{i}$. In more detail, let us consider in the same local card some curve $T_{i}(\eta)$ such that the following decomposition

$$
T_{i}=c_{i}+\eta L_{i}+o(\eta)
$$

holds true in the vicinity of the point $T_{i}=c_{i}$. Here $L_{i}$ are the coordinates in the tangent space.

Remark 1. The idea of linearization had come form the theory of Poisson-Lie groups, where $T_{i}$ are considered to be components of the group element and $L_{i}$ are considered to be components of the corresponding element of the Lie algebra. The expansion (8) in this case is simply expansion of the exponent in the Taylor power series. We will return to this case in the further examples. 
It occurred, that not for all the points $c \in M$ or, equivalently, not for all parameters $c_{i}$ such an expansion and "linearized" Poisson bracket exist. The following Proposition holds true:

Proposition 2.1 The expansion (8) define in a correct way a linear Poisson bracket on $V$ if and only if constants $c_{i}$ satisfy the following condition:

$$
\sum_{k, l \in I} C_{i j}^{k l} c_{k} c_{l}=0, \forall i, j \in I
$$

i.e. lie on zeros cone of the quadratic Poisson structure (7). The corresponding linear Poisson structure has in this case the following form:

$$
\left\{L_{i}, L_{j}\right\}_{1}=\sum_{k, l \in I} C_{i j}^{k l}\left(c_{k} L_{l}+c_{l} L_{k}\right)
$$

Proof. In order to obtain a correct linearization we will consider instead the bracket (7) an equivalent (proportional) bracket :

$$
\left\{T_{i}, T_{j}\right\}_{2}^{\prime}=\eta \sum_{k, l \in I} C_{i j}^{k l} T_{k} T_{l}
$$

Let us substitute expansion (8) in this Poisson bracket. Taking into account that $c_{i}$ are complex or real numbers constant lying in the kernel of the Poisson brackets we obtain:

$$
\eta^{2}\left\{L_{i}, L_{j}\right\}+o\left(\eta^{2}\right)=\eta \sum_{k, l \in I} C_{i j}^{k l} c_{k} c_{l}+\eta^{2} \sum_{k, l \in I} C_{i j}^{k l}\left(c_{k} L_{l}+c_{l} L_{k}\right)+o\left(\eta^{2}\right) .
$$

Comparing the coefficients near powers of $\eta$ in the both sides of this expression we obtain the statement of the Proposition.

Now, having defined the linear Poisson structure on the tangent space $V$, we can (using the diffeomorphism of the tangent space in a point of the local chart to the local chart itself) consider the bracket ( 7 ) also to be defined on the space $V$, i.e. on the coordinate functions $L_{i}$ or vise verse bracket (10) to be defined on the coordinate functions $T_{i}$.

In the subsequent we will use the following standard definition [1]:

Definition. Two poisson brackets $\{,\}_{2}$ and $\{,\}_{1}$ are called to be compatible if their linear combination is again a Poisson bracket.

The existence of the compatible Poisson bracket is important for the corresponding theory of integrable systems. The theory of compatible Poisson brackets was conceived in the paper [1] and developed in papers [2-7]. Using compatible Poisson brackets it is possible to construct the algebra of mutually Poisson-commutative functions - "integrals of motion" of an integrable hamiltonian system (see, for example, Theorem 2.1 below). 
The following important Proposition holds true:

Proposition 2.2 The quadratic order Poisson bracket (7) is always compatible with its linearization - the linear Poisson bracket (10).

Remark 2. Note that it is possible to define a linearization - the corresponding linear Poisson bracket for an arbitrary polynomial bracket of the degree $n$. But in the case $n>2$ the linearized bracket will not, generally speaking, be compatible with the initial polynomial Poisson bracket.

Proof. The statement of the Proposition follows from the combination of the previous Proposition and trick with the shift of the argument [24]. Let us consider shift of the local coordinates $T_{i}: T_{i} \rightarrow T_{i}+\eta c_{i}$, where $c_{i}$ are some constants and substitute it in the bracket (7). Using the fact that $c_{i}$ are constants we will obtain the following expression:

$$
\left\{T_{i}, T_{j}\right\}_{\eta} \equiv\left\{T_{i}+\eta c_{i}, T_{j}+\eta c_{j}\right\}_{2}=\sum_{k, l \in I}\left(C_{i j}^{k l} T_{k} T_{l}+\eta C_{i j}^{k l}\left(c_{k} T_{l}+c_{l} T_{k}\right)+\eta^{2} C_{i j}^{k l} c_{k} c_{l}\right) .
$$

Let now constants $c_{i}$ satisfy conditions (9) (this is needed to the very existence of the linearization (10)). In this case we will have that the last summand in the expression (12) vanishes and we obtain the following expression:

$$
\left\{T_{i}, T_{j}\right\}_{\eta}=\left\{T_{i}, T_{j}\right\}_{2}+\eta\left\{T_{i}, T_{j}\right\}_{1}
$$

where $\left\{T_{i}, T_{j}\right\}_{1} \equiv \sum_{k, l \in I} C_{i j}^{k l}\left(c_{k} T_{l}+c_{l} T_{k}\right)$ is "linearized" bracket (10) and $\left\{T_{i}, T_{j}\right\}_{2}=$ $\sum_{k, l \in I} C_{i j}^{k l} T_{k} T_{l}$ is the bracket (7). By other words, this means that linear combination of the brackets $\left\{T_{i}, T_{j}\right\}_{2}$ and $\left\{T_{i}, T_{j}\right\}_{1}$ is again Poisson bracket, i.e. these brackets are compatible.

Proposition is proved.

This Proposition is a generalization of the well-known in the quantum group theory fact about the compatibility of the "first and second Sklyanin brackets" [10]. In the next subsections we will consider several examples of this sort.

But at first, let us remind the reason of the importance of theory of compatible Poisson brackets (Lenard-Magri scheme) in the theory of classical integrable systems [1]. We will need the following version of this scheme of the construction of the set of mutually commuting functions starting from the Casimir functions of one of the compatible brackets:

Theorem 2.1 Let $I^{n}(T), I^{m}(T)$ be homogeneous polynomial invariants of the Poisson structure $\{,\}_{2}$ of the degree $n$ and $m$ correspondingly. Let $C$ satisfy conditions (10). Then the functions $I_{k}^{n}(T, C), I_{k}^{m}(T, C)$ obtained via the decomposition of the function $I^{n}(T+\eta C)$ and $I^{m}(T+\eta C)$ in the degrees of the parameter $\eta$ :

$$
I^{n}(T+\eta C)=\sum_{k=0}^{n} \eta^{k} I_{k}^{n}(T, C), I^{m}(T+\eta C)=\sum_{k=0}^{m} \eta^{k} I_{k}^{m}(T, C)
$$


constitute commuting family with respect to the brackets $\{,\}_{2}$ and $\{,\}_{1}$ :

$$
\left\{I_{k}^{n}(T, C), I_{l}^{m}(T, C)\right\}_{2}=\left\{I_{k}^{n}(T, C), I_{l}^{m}(T, C)\right\}_{1}=0 .
$$

Proof. In order to prove this Proposition let us note that $\left\{I^{n}(T+\eta C), F(T+\right.$ $\eta C)\}_{\eta}=0$ for arbitrary function $F$ due to the fact that $I^{n}(T+\eta C)$ is a Casimir function of the bracket $\{,\}_{\eta}$. Putting $F(T) \equiv I^{m}\left(T+\left(\eta^{\prime}-\eta\right) C\right)$ we obtain the following equality:

$$
\left\{I^{n}(T+\eta C), I^{m}\left(T+\eta^{\prime} C\right)\right\}_{\eta}=0 .
$$

In the analogous way one obtains the equality

$$
\left\{I^{n}(T+\eta C), I^{m}\left(T+\eta^{\prime} C\right)\right\}_{\eta^{\prime}}=0 .
$$

From these identities, decomposing the functions $I^{n}(T+\eta C)$ and $I^{m}\left(T+\eta^{\prime} C\right)$ in the powers of parameters $\eta$ and $\eta^{\prime}$ and using the fact that $\{,\}_{\eta}=\{,\}_{2}+\eta\{,\}_{1}$ we obtain the following equalities:

$$
\begin{aligned}
& \left\{I_{k}^{n}(T, C), I_{l}^{m}(T, C)\right\}_{2}+\left\{I_{k-1}^{n}(T, C), I_{l}^{m}(T, C)\right\}_{1}=0 . \\
& \left\{I_{k}^{n}(T, C), I_{l}^{m}(T, C)\right\}_{2}+\left\{I_{k}^{n}(T, C), I_{l-1}^{m}(T, C)\right\}_{1}=0,
\end{aligned}
$$

which are coefficients near the powers $\eta^{k} \eta^{\prime l}$ in these expansions. Taking into account that $I_{n}^{n}(T, C)=I^{n}(C), I_{m}^{m}(T, C)=I^{m}(C)$ are constants with respect to the both brackets, i.e.:

$$
\begin{aligned}
& \left\{I^{n}(C), I_{l}^{m}(T, C)\right\}_{2}=\left\{I^{n}(C), I_{l}^{m}(T, C)\right\}_{1}=0 \\
& \left\{I_{k}^{n}(T, C), I^{m}(C)\right\}_{2}=\left\{I_{k}^{n}(T, C), I^{m}(C)\right\}_{1}=0
\end{aligned}
$$

and substituting this into (15) we obtain the following equalities:

$$
\begin{gathered}
\left\{I_{n-1}^{n}(T, C), I_{l}^{m}(T, C)\right\}_{1}=0, l \geq 0, \\
\left\{I_{k}^{n}(T, C), I_{m-1}^{m}(T, C)\right\}_{1}=0, k \geq 0 .
\end{gathered}
$$

Making use of the equality (16a) and substituting it into the equality (15b), putting there $k=n-1$ we obtain that $\left\{I_{n-1}^{n}(T, C), I_{l}^{m}(T, C)\right\}_{2}=0, l>0$. In a similar way using the equality (16b), substituting it into the equality (15a), putting there $l=$ $m-1$ we obtain that $\left\{I_{m-1}^{m}(T, C), I_{k}^{n}(T, C)\right\}_{2}=0, k>0$. Taking into account that $I_{0}^{n}(T, C) \equiv I^{n}(T), I_{0}^{m}(T, C) \equiv I^{m}(T)$ are Casimir functions of the brackets $\{,\}_{2}$ we obtain also that $\left\{I_{n-1}^{n}(T, C), I_{0}^{m}(T, C)\right\}_{2}=0,\left\{I_{m-1}^{m}(T, C), I_{0}^{n}(T, C)\right\}_{2}=0$. Hence we have proved that

$$
\begin{aligned}
& \left\{I_{n-1}^{n}(T, C), I_{l}^{m}(T, C)\right\}_{2}=\left\{I_{n-1}^{n}(T, C), I_{l}^{m}(T, C)\right\}_{1}=0, l \in \overline{0, m} ; \\
& \left\{I_{m-1}^{m}(T, C), I_{k}^{n}(T, C)\right\}_{2}=\left\{I_{m-1}^{m}(T, C), I_{k}^{n}(T, C)\right\}_{1}=0, k \in \overline{0, n} .
\end{aligned}
$$


Proceeding inductively we at last obtain that

$$
\begin{aligned}
& \left\{I_{n-k}^{n}(T, C), I_{l}^{m}(T, C)\right\}_{1}=\left\{I_{k}^{n}(T, C), I_{m-l}^{m}(T, C)\right\}_{1}=0, \\
& \left\{I_{n-k}^{n}(T, C), I_{l}^{m}(T, C)\right\}_{2}=\left\{I_{k}^{n}(T, C), I_{m-l}^{m}(T, C)\right\}_{2}=0 .
\end{aligned}
$$

for $k \in \overline{0, n}, l \in \overline{0, m}$. Theorem is proved.

Remark 3. Note, that each point $C$ satisfying conditions (10) provides in the framework of our construction its own algebra of Poisson-commuting (with respect to the brackets $\left.\{,\}_{i}, i \in 1,2\right)$ integrals.

The following proposition (a kind of "Lenard recursive relation" [1]) is a consequence of the fact the constructed Poisson brackets $\{,\}_{i}, i \in 1,2$ are compatible:

Proposition 2.3 The hamiltonian equation of motion with respect to the bracket $\{,\}_{2}$ with the hamiltonian function $I_{k}^{n}(T, C),(k<n$ and is fixed) coincides with the hamiltonian equation of motion with respect to the brackets $\{,\}_{1}$ with the hamiltonian function $-I_{k-1}^{n}(T, C)$.

Proof. In order to prove this Proposition, let us consider the following Poisson bracket:

$$
\begin{gathered}
\left\{T_{i}+\eta C_{i}, I^{n}(T+\eta C)\right\}_{\eta}=\left\{T_{i}, I^{n}(T+\eta C)\right\}_{\eta}=\left\{T_{i}, I^{n}(T+\eta C)\right\}_{2}+ \\
+\eta\left\{T_{i}, I^{n}(T+\eta C)\right\}_{1}=\sum_{k=0}^{n} \eta^{k}\left\{T_{i}, I_{k}^{n}(T, C)\right\}_{2}+\sum_{k=1}^{n+1} \eta^{k}\left\{T_{i}, I_{k-1}^{n}(T, C)\right\}_{1}=0,
\end{gathered}
$$

where we have used that the function $I^{n}(T+\eta C)$ is a Casimir of the bracket $\{,\}_{\eta}$ and $C_{i}$ are constants with respect to this bracket. Due to the fact that this equality holds true for all degrees of the parameter $\eta$, we obtain for $0<k<n$ :

$$
\left\{T_{i}, I_{k}^{n}(T, C)\right\}_{2}+\left\{T_{i}, I_{k-1}^{n}(T, C)\right\}_{1}=0 .
$$

The hamiltonian equations of motion with respect to the hamiltonian $H$ and brackets $\{,\}_{k}$ are standardly defined as follows:

$$
\frac{d T_{i}}{d t}=\left\{T_{i}, H\right\}_{k}
$$

we obtain the statement of the proposition.

Remark 4. The above Proposition has a conceptual importance for the theory of classical dynamical systems. Indeed, it means that the integrable dynamical systems constructed with the help of quadratic Poisson brackets and the hamiltonian obtained with the help of the "argument shift method" as in the Theorem 2.1 may be interpreted as hamiltonian equations of motion with respect to the corresponding "linearized" Poisson bracket and the other "hamiltonian" obtained by the same 
"argument shift". Thus, on the level of classical integrable dynamical systems, it is sufficient to consider only linear Poisson brackets, because the corresponding quadratic bracket produces the same integrable dynamics.

\section{Quadratic Algebras and Poisson-Lie Groups}

In this section we will illustrate the results of the previous section on the examples of the quadratic Poisson algebra related to the RTT and to the Reflection Equation Algebras which are the main objects of interest of the present paper. In some cases the obtained results are well-known, in other cases they seemed to be new. For the sake of simplicity and in order to avoid global geometrical and topological subtleties we will restrict ourselves to the case of the Lie group $G L(n)$ and the corresponding Lie algebra $g l(n)$.

\subsection{First and Second Sklaynin Brackets}

\section{Compatibility}

Let us consider the reductive Lie algebra $g l(n)$ and its simple subalgebra $s l(n)$. Let us consider a meromorphic function of two complex variables $r_{12}(\lambda-\mu)$ taking values in $\operatorname{sl}(n) \otimes s l(n)$ satisfying the usual classical Yang-Baxter equation $[10,12,13]$ :

$$
\left[r_{12}(\lambda-\mu), r_{13}(\lambda-v)\right]=\left[r_{23}(\mu-v), r_{12}(\lambda-\mu)+r_{13}(\lambda-v)\right],
$$

where $r_{12}(\lambda-\mu)$, considered as a matrix acting in the space $s l(n)$, is nondegenerate.

Remark 5. It is possible also to consider nondegenerate $g l(n)$-valued $r$-matrices

$$
r^{\prime}(\lambda, \mu) \equiv f(\lambda, \mu) \cdot 1 \otimes 1+r(\lambda-\mu)
$$

where $r(\lambda-\mu) \in \operatorname{sl}(n) \otimes \operatorname{sl}(n), f(\lambda, \mu)$ is an arbitrary function of two complex variables, but they will lead to the same Poisson structures as $r(\lambda-\mu)$ and, that is why, we will restrict ourselves to the consideration of $s l(n)$-valued $r$-matrices.

The important fact [13] is that from the classical Yang-Baxter equation follows the skew-symmetry of the $r$-matrix $r_{12}(\lambda, \mu)$ :

$$
r_{12}(\lambda-\mu)=-r_{21}(\mu-\lambda)
$$

This gives a possibility to define, using $r_{12}(\lambda, \mu)$, so-called Sklyanin quadratic bracket [10]:

$$
\left\{T_{1}(\lambda), T_{2}(\mu)\right\}_{2}=\left[r_{12}(\lambda-\mu), T_{1}(\lambda) T_{2}(\mu)\right]
$$


where $T_{1}(\lambda) \equiv T(\lambda) \otimes 1, T_{2}(\mu) \equiv 1 \otimes T(\mu), T(\lambda)$ takes values in a classical matrix Lie group $G L(n)$, and is a meromorphic function of spectral parameter $\lambda$. Due to the fact, that all our consideration will have a local character, we will, slightly abusing the language, consider $T(\lambda)$ to be an element of $g l(n)$.

Remark 6. Generally speaking, the bracket (18) is defined in the infinitedimensional space of meromorphic functions of $\lambda$ where it defines a structure of an infinite-dimensional quadratic algebra. It also defines the structure of finitedimensional quadratic algebras in special subspaces of meromorphic functions. For example, in the case of $s l(2)$ elliptic $r$-matrix and matrices $T(\lambda)$ possessing one simple pole at $\lambda=0$ it defines the Sklyanin algebra [25] or "many-poled" Sklyanin algebras in the cases of matrices $T(\lambda)$ with many simple poles [15].

We will not need to write more explicitly the commutation relations (18) leaving them in the "convoluted" form. Let us now consider the expansion (8):

$$
T(\lambda)=C(\lambda)+\eta L(\lambda)+o(\eta),
$$

where $C \in G L(n)$. It gives us the following linearization:

$$
\left\{L_{1}(\lambda), L_{2}(\mu)\right\}_{1}=\left[r_{12}(\lambda-\mu), L(\lambda) \otimes C(\mu)+C(\lambda) \otimes L(\mu)\right] .
$$

Here the "initial point" $C$ in this decomposition satisfies the following condition:

$$
\left[r_{12}(\lambda, \mu), C(\lambda) \otimes C(\mu)\right]=0 .
$$

Remark 7. Note that the linearization condition (20) has the same form for all infinite-dimensional quadratic structures defined by the (18) and for all finitedimensional substructures (like the Sklyanin algebra [25], the many-poled Sklyanin algebra [15], etc.).

In the special case, when $C(\lambda)$ coincides with a unit element of the group $G L(n)$ $(C(\lambda)=1)$, we obtain from (21) the standard "second" Sklyanin bracket:

$$
\left\{L_{1}(\lambda), L_{2}(\mu)\right\}_{1}=\left[r_{12}(\lambda-\mu), L_{1}(\lambda)+L_{2}(\mu)\right] .
$$

Hence we have recovered the well-known fact [11] that "first" and "second Sklyanin brackets" are compatible.

It is easy to show that the bracket (19) is equivalent to the bracket (21). Indeed, making a change of variables: $L(\lambda)=L^{C}(\lambda) C(\lambda)$ and using the condition (19) we obtain that the bracket (19) pass to the bracket (21). Hence, in this class of examples one does not come to the different compatible structures choosing different initial points for linearization. The situation is different in the case of other quadratic algebras, the examples of which we will consider in the next subsections. As we will see the choice of the "initial point" in the neighborhood of which we linearize Poisson bracket may lead to different Lie algebraic structures. 


\section{Algebra of Integrals}

Let us now consider an impact of compatibility of quadratic and linear Sklyanin brackets in the corresponding theory of integrable systems. We will show, in particular, that commutativity of generating functions of the classical integrals $\operatorname{tr}(T(\lambda))^{k}$ (or $\operatorname{tr}(L(\lambda))^{k}$ ) with respect to the both linear and second order Poisson brackets may be derived using only the theory of compatible Poisson brackets.

In order to use the theory of bihamiltonian systems we have to describe Casimir functions of the quadratic Sklyanin brackets on $g l(n)$. The following result is true:

Theorem 3.1 Let $d(\lambda)$ be a determinant of the $g l(n)$-valued monodromy matrix $T(\lambda)$. Then $d(\lambda)$ is a generating function of the Casimir functions of the bracket (18) for all types of "monodromy" matrices $T(\lambda)$ satisfying the brackets (18) and for all types of $\operatorname{sl}(n) \otimes s l(n)$-valued $r$-matrices.

Proof. In order to prove the theorem we have to show that $\left\{d(\lambda), T_{i j}(\mu)\right\}_{2}=0$, where the bracket $\{,\}_{2}$ has the form (18). Let us notice at first that $\operatorname{tr} L(\lambda)$ is a Casimir function of the bracket (21) for any $s l(n)$-valued $r$-matrix $r_{12}(\lambda, \mu)$. Indeed:

$$
\left\{\operatorname{tr}_{1}\left(L_{1}(\lambda)\right), L_{2}(\mu)\right\}=\operatorname{tr}_{1}\left(\left[r_{12}(\lambda-\mu), L_{1}(\lambda)\right]\right)+\left[\operatorname{tr}_{1}\left(r_{12}(\lambda-\mu)\right), L_{2}(\mu)\right]=0 .
$$

The first summand is equal to zero as a trace of commutator and the second one is equal to zero because $\operatorname{tr}_{1}\left(r_{12}(\lambda-\mu)\right)=0$ due to the fact that our $r$-matrix is $s l(n)$-valued.

Now let us make the following change of variables:

$$
T(\lambda)=\exp L(\lambda) .
$$

Keeping in mind the well-known relation of determinants and traces we obtain:

$$
d(\lambda) \equiv \operatorname{det} T(\lambda)=\exp \operatorname{tr} L(\lambda)
$$

If the change of variables (22) had been Poisson,the equality (23) would have been sufficient for the proof of the theorem. But, unfortunately, it is not Poisson. Nevertheless the change of variables (22) will be still useful. Indeed, let us explicitly calculate expressions $\left\{\exp L_{1}(\lambda), \exp L_{2}(\mu)\right\}_{2}$ and $\left\{\exp L_{1}(\lambda), \exp L_{2}(\mu)\right\}_{1}$. We have:

$$
\begin{aligned}
& \left\{\exp L_{1}(\lambda), \exp L_{2}(\mu)\right\}_{2}=\left[r_{12}(\lambda-\mu), \exp L_{1}(\lambda) \exp L_{2}(\mu)\right]= \\
& \quad=\sum_{k, l=0}^{\infty} \frac{1}{k ! l !}\left[r_{12}(\lambda-\mu), L_{1}^{k}(\lambda) L_{2}^{l}(\mu)\right] .
\end{aligned}
$$

Hereafter it is implied that $L_{1}^{0}(\lambda) \equiv 1$. On the other hand we obtain:

$$
\left\{\exp L_{1}(\lambda), \exp L_{2}(\mu)\right\}_{1}=\sum_{k, l=0}^{\infty} \frac{1}{k ! l !}\left\{L_{1}^{k}(\lambda), L_{2}^{l}(\mu)\right\}_{1} .
$$


By the recursion applied first to $k$ and then to $l$ we obtain the following equality:

$$
\begin{aligned}
\left\{L_{1}^{k}(\lambda), L_{2}^{l}(\mu)\right\}_{1}=\left(\sum_{i=0}^{k-1} L_{1}(\lambda)^{i}\left[r_{12}(\lambda-\mu), L_{2}^{l}(\mu)\right] L_{1}(\lambda)^{k-1-i}+\right. \\
\\
\left.\sum_{j=0}^{l-1} L_{2}(\mu)^{j}\left[r_{12}(\lambda-\mu), L_{1}^{k}(\lambda)\right] L_{2}(\mu)^{l-1-j}\right) .
\end{aligned}
$$

Now we will rewrite the formula (26) in the following way:

$$
\begin{gathered}
\frac{1}{k ! l !}\left\{L_{1}^{k}(\lambda), L_{2}^{l}(\mu)\right\}_{1}= \\
\frac{1}{2(k-1) ! l !}\left(L_{1}(\lambda)^{k-1}\left[r_{12}(\lambda-\mu), L_{2}^{l}(\mu)\right]+\left[r_{12}(\lambda-\mu), L_{2}^{l}(\mu)\right] L_{1}(\lambda)^{k-1}\right)+ \\
\frac{1}{2 k !(l-1) !}\left(\left[r_{12}(\lambda-\mu), L_{1}^{k}(\lambda)\right] L_{2}(\mu)^{l-1}+L_{2}(\mu)^{l-1}\left[r_{12}(\lambda-\mu), L_{1}^{k}(\lambda)\right]\right)+ \\
\frac{1}{2 k ! l !}\left(\sum_{i=0}^{k-1}\left[L_{1}(\lambda)^{i},\left[\left[r_{12}(\lambda-\mu), L_{2}^{l}(\mu)\right], L_{1}(\lambda)^{k-1-i}\right]\right]+\right. \\
\left.\sum_{j=0}^{l-1}\left[\left[L_{2}(\mu)^{j},\left[r_{12}(\lambda-\mu), L_{1}^{k}(\lambda)\right]\right], L_{2}(\mu)^{l-1-j}\right]\right) .
\end{gathered}
$$

Using this equality, the Leibnitz rule for the commutator, the fact that $\left[L_{1}^{k}(\lambda)\right.$, $\left.L_{2}^{l}(\mu)\right]=0$ and renaming the indices $k-1 \rightarrow k$, when summing the first two summands of the right-hand side of this equality with respect to $k, l$ and $l-1 \rightarrow l$ when summing the next two summands of the right-hand side of this equality with respect to $k, l$, it is easy to show that

$$
\begin{gathered}
\sum_{k, l=0}^{\infty} \frac{1}{k ! l !}\left\{L_{1}^{k}(\lambda), L_{2}^{l}(\mu)\right\}_{1}=\sum_{k, l=0}^{\infty} \frac{1}{k ! l !}\left[r_{12}(\lambda-\mu), L_{1}^{k}(\lambda) L_{2}^{l}(\mu)\right]+ \\
\sum_{k, l=0}^{\infty} \frac{1}{2 k ! l !}\left(\sum_{i=0}^{k-1}\left[L_{1}(\lambda)^{i},\left[\left[r_{12}(\lambda-\mu), L_{2}^{l}(\mu)\right], L_{1}(\lambda)^{k-1-i}\right]\right]+\right. \\
\left.\sum_{j=0}^{l-1}\left[\left[L_{2}(\mu)^{j},\left[r_{12}(\lambda-\mu), L_{1}^{k}(\lambda)\right]\right], L_{2}(\mu)^{l-1-j}\right]\right) .
\end{gathered}
$$

By other words we have obtained that:

$$
\left\{\exp L_{1}(\lambda), \exp L_{2}(\mu)\right\}_{2}=\left\{\exp L_{1}(\lambda), \exp L_{2}(\mu)\right\}_{1}-\tilde{X}_{12}(L), \text { where }
$$




$$
\begin{aligned}
\tilde{X}_{12}(L) & \equiv \sum_{k, l=0}^{\infty} \frac{1}{2 k ! l !}\left(\sum_{i=0}^{k-1}\left[L_{1}(\lambda)^{i},\left[\left[r_{12}(\lambda-\mu), L_{2}^{l}(\mu)\right], L_{1}(\lambda)^{k-i}\right]\right]+\right. \\
& \left.+\sum_{j=0}^{l-1}\left[\left[L_{2}(\mu)^{j},\left[r_{12}(\lambda-\mu), L_{1}^{k}(\lambda)\right]\right], L_{2}(\mu)^{l-1-j}\right]\right) .
\end{aligned}
$$

Now, we remark, that using the Jacobi identity and the fact that $\left[L_{1}^{k}(\lambda), L_{2}^{j}(\mu)\right]=0$ the expression $\left.\sum_{j=0}^{l-1}\left[\left[L_{2}(\mu)^{j},\left[r_{12}(\lambda-\mu), L_{1}^{k}(\lambda)\right]\right], L_{2}(\mu)^{l-1-j}\right]\right)$ can be further transformed in the following form: $\sum_{j=0}^{l-1}\left[L_{1}(\lambda)^{k},\left[\left[r_{12}(\lambda-\mu), L_{2}^{j}(\mu)\right], L_{2}(\mu)^{l-1-j}\right]\right]$. Hence, we can re-write the expression for $\widetilde{X}_{12}(L)$ as:

$$
\tilde{X}_{12}(L)=\sum_{m=0}^{\infty}\left[L_{1}^{m}(\lambda), X_{12}^{(m)}(L)\right]
$$

Now, let us consider the expression

$$
\begin{aligned}
\{d(\lambda), & \left.T_{2}(\mu)\right\}_{2} \equiv \sum_{k, l=1}^{n}\left\{d(\lambda), T_{k l}(\mu)\right\}_{2} X_{k l}= \\
& =\sum_{i, j, k, l=1}^{n} \frac{\partial d(\lambda)}{\partial T_{i j}(\lambda)}\left\{T_{i j}(\lambda), T_{k l}(\mu)\right\}_{2} X_{k l}=\left\langle\nabla_{1} d(\lambda),\left\{T_{1}(\lambda), T_{2}(\mu)\right\}_{2}\right\rangle_{1},
\end{aligned}
$$

where $\nabla_{1} d(\lambda) \equiv \sum_{i, j=1}^{n} \frac{\partial d(\lambda)}{\partial T_{i j}(\lambda)} X_{j i} \otimes 1$, and $\langle,\rangle_{1}$ means the scalar product in the first factor of the tensor product $g l(n) \otimes g l(n)$. As it was shown above, we can re-write this as follows:

$$
\left\langle\nabla_{1} d(\lambda),\left\{T_{1}(\lambda), T_{2}(\mu)\right\}_{2}\right\rangle_{1}=\left\langle\nabla_{1} d(\lambda),\left\{T_{1}(\lambda), T_{2}(\mu)\right\}_{1}\right\rangle_{1}-\left\langle\nabla_{1} d(\lambda), \widetilde{X}_{12}(L)\right\rangle_{1},
$$

where $\left\{T_{1}(\lambda), T_{2}(\mu)\right\}_{1} \equiv\left\{\exp L_{1}(\lambda), \exp L_{2}(\mu)\right\}_{1}$. On the other hand first summand in (28) can be written in the following form:

$$
\left\langle\nabla_{1} d(\lambda),\left\{T_{1}(\lambda), T_{2}(\mu)\right\}_{1}\right\rangle_{1}=\left\{d(\lambda), T_{2}(\mu)\right\}_{1}=\left\{\exp \operatorname{tr} L_{1}(\lambda), \exp L_{2}(\mu)\right\}_{1} .
$$

Taking into account that $\operatorname{tr} L(\lambda)$ is a Casimir function of the brackets $\{,\}_{1}$ we obtain that $\left\{\exp \operatorname{tr} L_{1}(\lambda), \exp L_{2}(\mu)\right\}_{1}=0$.

Let us consider the second summand in the equality (28). Let us take into account that $\frac{1}{d(\lambda)} \frac{\partial d(\lambda)}{\partial T_{i j}(\lambda)}=\left(T^{-1}(\lambda)\right)_{j i}$ and, hence, $\nabla_{1} d(\lambda)=d(\lambda) T_{1}^{-1}(\lambda)$. That is why 
we obtain:

$$
\left\langle\frac{\nabla_{1} d(\lambda)}{d(\lambda)}, \widetilde{X}_{12}(L)\right\rangle_{1}=\left\langle T_{1}^{-1}(\lambda), \widetilde{X}_{12}(L)\right\rangle_{1}=\sum_{m=0}^{\infty}\left\langle T_{1}(\lambda)^{-1},\left[L_{1}^{m}(\lambda), X_{12}^{(m)}(L)\right]\right\rangle_{1} .
$$

On the other hand

$$
\left\langle T_{1}(\lambda)^{-1},\left[L_{1}^{m}(\lambda), X_{12}^{(m)}(L)\right]\right\rangle_{1}=\left\langle\left[T_{1}(\lambda)^{-1}, L_{1}^{m}(\lambda)\right], X_{12}^{(m)}(L)\right\rangle_{1},
$$

where we have used the $a d$-invariance of the pairing $\langle,\rangle_{1}$. Now using the fact that $T_{1}(\lambda)^{-1}=\exp \left(-L_{1}(\lambda)\right)$ and the evident fact that $\left[\exp \left(-L_{1}(\lambda)\right), L_{1}^{m}(\lambda)\right]=0$ we obtain that the second summand in (28) is also zero.

Theorem is proved.

Remark 8. The theorem above is a generalization of the well-known for the concrete $r$-matrices fact that $\operatorname{det} T(\lambda)$ is a Casimir function of the quadratic $r$-matrix bracket onto the case of the arbitrary $r$-matrices (including all trigonometric $r$-matrices of Belavin and Drienfield [13], non-standard rational $r$-matrices of Stolin [26], etc.).

From the Theorems 3.1 and 2.1 follows the next:

Corollary 3.1 Denote by $d_{k}(T(\lambda))$ the coefficients in the decomposition: $\operatorname{Det}(T(\lambda)+$ $\eta I)=\sum_{k=0}^{n} d_{k}(T(\lambda)) \eta^{k}$. Then $d_{k}(T(\lambda))$ are generators of an Abelian subalgebra with respect to the both linear and quadratic r-matrix brackets (21) and (18), i.e.:

$$
\left\{d_{k}(T(\lambda)), d_{l}(T(\mu))\right\}_{1}=\left\{d_{k}(T(\lambda)), d_{l}(T(\mu))\right\}_{2}=0 .
$$

Proof. In order to prove this corollary it is enough to use the Theorem 2.1 applied for the two polynomial Casimir functions $d(T(\lambda))$ and $d(T(\mu))$ of the bracket $\{,\}_{2}$.

Remark 9. Functions $\left\{d_{k}(T(\lambda))\right\}$ define the same algebra of integrals as functions $\left\{\operatorname{tr} T^{l}(\lambda)\right\}$ and are connected with them by the well-known Newton formulas. Nevertheless, it is nice to notice that they could be obtained from the consideration of bihamiltonity and Casimir element $d(\lambda)$ only. This may have deep meaning, because in the quantum case operators $\left\{\operatorname{tr} \hat{T}^{l}(\lambda)\right\}$ stops to be commutative, but quantum analogues of the functions $\left\{d_{k}(T(\lambda))\right\}$ still are [27,28].

Remark 10. It is easy to see that integrals obtained by the shift on another matrix $C \in G l(n)$ are equivalent to the integrals obtained by the shift on the unit matrix. Indeed, it is evident that $\operatorname{Det}(T(\lambda)+\eta C(\lambda))=\operatorname{Det} C \operatorname{Det}\left(T^{C}(\lambda)+\eta I\right)$, where $T^{C}(\lambda)=T(\lambda) C^{-1}(\lambda)$. But, due to the fact that $\left[r_{12}(\lambda, \mu), C(\lambda) \otimes C(\mu)\right]=$ $\left[r_{12}(\lambda, \mu), C^{-1}(\lambda) \otimes C^{-1}(\mu)\right]=0$ elements $T^{C}(\lambda)$ define the same algebra (both with respect to the quadratic and the linear brackets) as elements $T(\lambda)$ in the case $C(\lambda)=1$

Now, let us consider what the condition of compatibility of brackets (21) and (18) yields from the point of view of the corresponding dynamics. For this purpose, in 
principle, we need to decompose the generating functions $d_{k}(T(\lambda))$ with respect to the spectral parameter $\lambda$. Using the fact that $T(\lambda)$ is meromorphic functions this can always be done using the decomposition in Laurent power series. In the special cases the other decompositions may be also used [14]. In the present paper we will not do these decompositions explicitly leaving all the expressions in the "convoluted" form of the generating functions. Using the Proposition 2.3 we obtain the following:

Proposition 3.1 Hamiltonian equations of motion with respect to the bracket $\{,\}_{2}$ with a hamiltonian being one of the functions obtained by a decomposition of function $d_{k}(T(\lambda)), k<n$, coincide with the hamiltonian equations of motion with respect to the brackets $\{,\}_{1}$ with the hamiltonian being the corresponding function obtained by the decomposition of function $-d_{k-1}(T(\lambda))$, i.e. on the level of generating functions the following equality is true:

$$
\left\{d_{k}(T(\lambda)), T(\mu)\right\}_{2}=-\left\{d_{k-1}(T(\lambda)), T(\mu)\right\}_{1} .
$$

In the case $k=n-1$ this Proposition implies the following important corollary:

Corollary 3.2 Hamiltonian equations of motion with respect to the bracket $\{,\}_{2}$ with a hamiltonian being one of the functions obtained by a decomposition of function $\operatorname{tr} T(\lambda)$ coincide with the hamiltonian equations of motion with respect to the brackets $\{,\}_{1}$ with the hamiltonian being the corresponding function obtained by the decomposition of the function $\frac{1}{2} \operatorname{tr}\left(T^{2}(\lambda)\right)$, i.e. on the level of generating functions the following equality holds true:

$$
\{\operatorname{tr}(T(\lambda)), T(\mu)\}_{2}=\frac{1}{2}\left\{\operatorname{tr}\left(T^{2}(\lambda)\right), T(\mu)\right\}_{1} .
$$

Proof. In order to derive this statement it is sufficient to put in the previous proposition $k=n-1$, use Newton identities and the fact that function $\operatorname{tr} T(\lambda)$ is a Casimir function of the linear $r$-matrix bracket $\{,\}_{1}$.

\section{2 “Twisted Reflection Equation Algebra” and Its Linearizations}

\section{Compatible Brackets}

Let, as in the previous subsection, $r(\lambda-\mu)$ be a $s l(n) \otimes \operatorname{sl}(n)$-valued solution of the classical Yang-Baxter equation (17), and $\mathcal{T}(\lambda)$ be a meromorphic function of spectral parameter $\lambda$ taking the values in a classical matrix Lie group $G L(n)$.

Let $\sigma$ be some automorphism of $g l(n)$ (and $s l(n)$ ). Let us consider the space of functions $\mathcal{T}(\lambda)$ the following quadratic brackets:

$$
\begin{aligned}
\left\{\mathcal{T}_{1}(\lambda), \mathcal{T}_{2}(\mu)\right\}_{2}= & r_{12}(\lambda-\mu) \mathcal{T}_{1}(\lambda) \mathcal{T}_{2}(\mu)-\mathcal{T}_{1}(\lambda) r_{12}^{\sigma_{1}}(-\lambda-\mu) \mathcal{T}_{2}(\mu)- \\
& -\mathcal{T}_{2}(\mu) r_{12}^{\sigma_{2}}(\lambda+\mu) \mathcal{T}_{1}(\lambda)+\mathcal{T}_{1}(\lambda) \mathcal{T}_{2}(\mu) r_{12}^{\sigma_{1} \sigma_{2}}(\mu-\lambda),
\end{aligned}
$$


where $r_{12}^{\sigma_{1}}(\lambda)=\sigma \otimes 1 \cdot r_{12}(\lambda), r_{12}^{\sigma_{2}}(\lambda)=1 \otimes \sigma \cdot r_{12}(\lambda)$, etc. By direct and tedious calculation one can show that (29) is indeed a Poisson bracket, i.e. that it satisfies Jacobi condition for the case of arbitrary automorphism $\sigma$ and an arbitrary solution $r_{12}(\lambda-\mu)$ of the classical Yang-Baxter equation (see also Remark 14).

One may consider the bracket (29) defined on the infinite-dimensional space of meromorphic functions of $\lambda$ like in the case of previous subsection. We will call the corresponding algebra a "twisted classical Reflection Equation Algebra". The bracket (29) may be also considered in some special finite-dimensional subspaces of the space of meromorphic functions where it defines a structure of a finitedimensional quadratic algebra (see for example [16]).

Remark 11. Due to the known fact that all automorphisms of $g l(n)$ are either internal or have a second order we may write that $\sigma=A d_{K_{0}} \sigma_{0}$, where $K_{0} \in G L(n)$ and $\sigma_{0}$ is an involutive external automorphism of $g l(n)$ or $\sigma_{0}=1$. All external automorphisms of $g l(n)$ as a Lie algebra are can be written as minus anti-automorphism of $g l(n)$ considered as an associative algebra, and we may write symbolically that $\sigma_{0}(X)=-X^{t}$ where upper superscript $t$ denotes anti-automorphism of $g l(n)$ (in particular the ordinary transposition). Moreover by substitution $\mathcal{T}(\lambda) \rightarrow \mathcal{T}(\lambda) K_{0}^{-1}$ we can get rid of $A d_{K_{0}}$ and to consider hereafter only the case $\sigma=\sigma_{0}$.

In the simplest case $\sigma=1$, using the skew-symmetry of $r(\lambda-\mu)$, we obtain the "classical Reflection Equation Algebra" which is a classical limit of the standard quantum Reflection Equation Algebra [10,19]:

$$
\begin{array}{r}
\left\{\mathcal{T}_{1}(\lambda), \mathcal{T}_{2}(\mu)\right\}_{2}=r_{12}(\lambda-\mu) \mathcal{T}_{1}(\lambda) \mathcal{T}_{2}(\mu)+\mathcal{T}_{1}(\lambda) r_{21}(\lambda+\mu) \mathcal{T}_{2}(\mu)- \\
-\mathcal{T}_{2}(\mu) r_{12}(\lambda+\mu) \mathcal{T}_{1}(\lambda)-\mathcal{T}_{1}(\lambda) \mathcal{T}_{2}(\mu) r_{21}(\lambda-\mu) .
\end{array}
$$

Let us linearize the quadratic bracket (29) it in the neighborhood of some point $\mathcal{T}(\lambda)=K(\lambda)$. The sufficient condition of an existence of such a linearization is the requirement (9), which acquires in the case at hand the following form:

$$
\begin{aligned}
& r_{12}(\lambda-\mu) K_{1}(\lambda) K_{2}(\mu)+K_{1}(\lambda) K_{2}(\mu) r_{12}^{\sigma_{1} \sigma_{2}}(\mu-\lambda)= \\
& =K_{2}(\mu) r_{12}^{\sigma_{2}}(\lambda+\mu) K_{1}(\lambda)+K_{1}(\lambda) r_{12}^{\sigma_{1}}(-\lambda-\mu) K_{2}(\mu) .
\end{aligned}
$$

Remark 12. For the case $\sigma=1$ and $r$ matrices $r_{12}(\lambda-\mu)$ satisfying a symmetry condition:

$$
r_{12}(\lambda-\mu)=r_{21}(\lambda-\mu),
$$

the simplest possible choice $K(\lambda)=1$ is a solution of (31). In $s l(2)$ case the condition (32) is true, for example, for the classical rational $r$-matrix of Yang, for standard trigonometric $r$-matrix and elliptic $r$-matrix. It is not true for non-standard rational $r$-matrices of Stolin. In the case $s l(n), n>2$ condition (32) is not true nor for standard trigonometric $r$-matrices neither for $s l(n)$ elliptic $r$-matrices. Moreover, as we will show in the example below, even for the $r$-matrices for which condition (32) is satisfied there are a lot of other (except $K=1$ ) solutions of (31). 
Using nondegenerate matrices $K(\lambda)$ satisfying condition (31) it is possible to introduce the corresponding linear bracket (10) which will have the following explicit form:

$$
\begin{aligned}
& \quad\left\{\mathcal{L}_{1}(\lambda), \mathcal{L}_{2}(\mu)\right\}_{1}=r_{12}(\lambda-\mu) K_{1}(\lambda) \mathcal{L}_{2}(\mu)+r_{12}(\lambda-\mu) \mathcal{L}_{1}(\lambda) K_{2}(\mu)- \\
& K_{1}(\lambda) r_{12}^{\sigma_{1}}(-\lambda-\mu) \mathcal{L}_{2}(\mu)-\mathcal{L}_{2}(\mu) r_{12}^{\sigma_{2}}(\lambda+\mu) K_{1}(\lambda)+K_{1}(\lambda) \mathcal{L}_{2}(\mu) r_{12}^{\sigma_{1} \sigma_{2}}(\mu-\lambda)- \\
& -\mathcal{L}_{1}(\lambda) r_{12}^{\sigma_{1}}(-\lambda-\mu) K_{2}(\mu)-K_{2}(\mu) r_{12}^{\sigma_{2}}(\lambda+\mu) \mathcal{L}_{1}(\lambda)-\mathcal{L}_{1}(\lambda) K_{2}(\mu) r_{12}^{\sigma_{1} \sigma_{2}}(\mu-\lambda) .
\end{aligned}
$$

Despite its complicated form, bracket (33) may be substantially simplified. Indeed, after the replacement of the variables: $\mathcal{L}(\lambda)=\mathcal{L}^{K}(\lambda) K(\lambda)$, usage of the equality (31) and skew symmetry of $r_{12}(\lambda-\mu)$ it can be re-written in the following simple form:

$$
\begin{aligned}
\left\{\mathcal{L}_{1}^{K}(\lambda), \mathcal{L}_{2}^{K}(\mu)\right\}_{1}= & {\left[r_{12}(\lambda-\mu)-K_{2}(\mu) r_{12}^{\sigma_{2}}(\lambda+\mu) K_{2}^{-1}(\mu), \mathcal{L}_{1}^{K}(\lambda)\right]-} \\
& -\left[r_{21}(\mu-\lambda)-K_{1}(\lambda) r_{21}^{\sigma_{1}}(\lambda+\mu) K_{1}^{-1}(\lambda), \mathcal{L}_{2}^{K}(\mu)\right] .
\end{aligned}
$$

Linear Poisson bracket (34) is a particular example of linear Poisson brackets governed by the non-skew symmetric $r$-matrices $r_{12}(\lambda, \mu)$ :

$$
\left\{L_{1}(\lambda), L_{2}(\mu)\right\}_{1}=\left[r_{12}(\lambda, \mu), L_{1}(\lambda)\right]-\left[r_{21}(\mu, \lambda), L_{2}(\mu)\right],
$$

where $r_{12}(\lambda, \mu)$ is a solution of "generalized" classical Yang-Baxter equation [20-22]:

$$
\left[r_{12}(\lambda, \mu), r_{13}(\lambda, v)\right]=\left[r_{23}(\mu, v), r_{12}(\lambda, \mu)\right]+\left[r_{13}(\lambda, v), r_{32}(v, \mu)\right] .
$$

As it is evident from the very definition, in our case:

$$
r_{12}(\lambda, \mu)=r_{12}^{\sigma, K}(\lambda, \mu) \equiv r_{12}(\lambda-\mu)-K_{2}(\mu) r_{12}^{\sigma_{2}}(\lambda+\mu) K_{2}^{-1}(\mu)
$$

Remark 13. The classical $r$-matrix (36) can be rewritten in the form:

$$
r_{12}^{\sigma(\mu)}(\lambda, \mu)=r_{12}(\lambda-\mu)-\sigma_{2}(\mu) \cdot r_{12}(\lambda+\mu),
$$

where $\sigma_{2}(\mu) \equiv A d_{K_{2}(\mu)} \cdot \sigma_{2}$. The $r$-matrix (37) may be obtained also from the other considerations without appealing to the "twisted" quadratic Poisson brackets (29) (see [23]).

Hence, we see that in this case the resulting classical $r$-matrices $r_{12}^{\sigma, K}(\lambda, \mu)$ and corresponding linear Lie-algebraic structures substantially depend on the initial point $K(\lambda)$ around which we linearize our quadratic Poisson bracket. For the fixed $r$-matrix $r(\lambda-\mu)$ there may be many (even parametric families!) of such the points $K(\lambda)$ and all of the corresponding linear Poisson structures are compatible with 
the quadratic Poisson algebra (29). In order to illustrate this, we will consider the following example of such the points $K(\lambda)$ and their $r$-matrices $r_{12}^{\sigma, K}(\lambda, \mu)$ :

Example 1. Let $\mathfrak{g}=\operatorname{sl}(2)$ and $\sigma=1$. Let $X_{i}, i \in 1,3$ be the orthonormal basis in $\operatorname{sl}(2) \simeq \operatorname{so}(3)$ with the commutation relations:

$$
\left[X_{i}, X_{j}\right]=\epsilon_{i j k} X_{k}
$$

Let us consider the classical elliptic $r$-matrix of Sklyanin [18]:

$$
r(\lambda-\mu)=\sum_{k=1}^{3} r_{k}(\lambda-\mu) X_{k} \otimes X_{k}
$$

where $r_{k}(\lambda)$ are expressed via Jacobi functions:

$$
r_{1}(\lambda)=\frac{1}{\operatorname{sn}(\lambda)}, r_{2}(u)=\frac{d n(\lambda)}{\operatorname{sn}(\lambda)}, r_{3}(u)=\frac{\operatorname{cn}(\lambda)}{\operatorname{sn}(\lambda)} .
$$

It is easy to see that $r_{12}(\lambda-\mu)=-r_{12}(\lambda-\mu)$ due to the fact that functions $r_{k}(\lambda)$ are odd.

Let us now introduce the standard "root" basis in $\operatorname{sl}(2): X_{0}=i X_{3}, X_{ \pm}=i\left(X_{1} \pm\right.$ $i X_{2}$ ), with the standard commutation relations:

$$
\left[X_{0}, X_{ \pm}\right]= \pm X_{ \pm},\left[X_{+}, X_{-}\right]=2 X_{0}
$$

In this basis we have that the skew-symmetric elliptic $r$-matrix acquires the following form:

$$
\begin{aligned}
r(\lambda-\mu)=r_{0}(\lambda-\mu) X_{0} \otimes X_{0}+ & r_{+}(\lambda-\mu)\left(X_{+} \otimes X_{-}+X_{-} \otimes X_{+}\right)+ \\
& +r_{-}(\lambda-\mu)\left(X_{+} \otimes X_{+}+X_{-} \otimes X_{-}\right),
\end{aligned}
$$

where $r_{0}(\lambda)=r_{3}(\lambda), r_{+}(\lambda)=\frac{1}{4}\left(r_{2}(\lambda)+r_{1}(\lambda)\right), r_{-}(\lambda)=\frac{1}{4}\left(r_{2}(\lambda)-r_{1}(\lambda)\right)$ and $r_{i}(\lambda)$ are defined using the formula (39).

Using the addition laws for the Jacobi functions [29] it is possible to prove that the matrix

$$
K(\lambda) \equiv K(\lambda, \xi)=\operatorname{diag}\left(k_{1}(\lambda), k_{2}(\lambda)\right) \equiv \operatorname{diag}\left(\frac{\operatorname{sn}(\lambda)}{\operatorname{cn}(\lambda)}+\frac{\operatorname{sn}(\xi)}{\operatorname{cn}(\xi)},-\frac{\operatorname{sn}(\lambda)}{\operatorname{cn}(\lambda)}+\frac{\operatorname{sn}(\xi)}{\operatorname{cn}(\xi)}\right)
$$

satisfies (31) for arbitrary complex parameter $\xi$. This permits one to define a new non-skew symmetric elliptic $r$-matrix using the formula (36). It has the following form:

$$
\begin{aligned}
r^{K}(\lambda, \mu)=r_{0}^{K}(\lambda, \mu) X_{0} \otimes & X_{0}+r_{+-}^{K}(\lambda, \mu) X_{+} \otimes X_{-}+r_{-+}^{K}(\lambda, \mu) X_{-} \otimes X_{+}+ \\
& +r_{++}^{K}(\lambda, \mu) X_{+} \otimes X_{+}+r_{--}^{K}(\lambda, \mu) X_{-} \otimes X_{-},
\end{aligned}
$$


where the correspondent coefficients are given by the following formulas:

$$
\begin{gathered}
r_{+-}^{K}(\lambda, \mu)=r_{+}(\lambda-\mu)-\frac{k_{2}(\mu)}{k_{1}(\mu)} r_{+}(\lambda+\mu), \\
r_{-+}^{K}(\lambda, \mu)=r_{+}(\lambda-\mu)-\frac{k_{1}(\mu)}{k_{2}(\mu)} r_{+}(\lambda+\mu), \\
r_{++}^{K}(\lambda, \mu)=r_{-}(\lambda-\mu)-\frac{k_{1}(\mu)}{k_{2}(\mu)} r_{-}(\lambda+\mu), \\
r_{--}^{K}(\lambda, \mu)=r_{-}(\lambda-\mu)-\frac{k_{2}(\mu)}{k_{1}(\mu)} r_{-}(\lambda+\mu), \\
r_{0}^{K}(\lambda, \mu)=r_{0}(\lambda-\mu)-r_{0}(\lambda+\mu) .
\end{gathered}
$$

\section{Algebra of Integrals}

Let us now consider the impact that has a compatibility of the second and linear Poisson brackets on the corresponding theory of the integrable systems. We will show, in particular, that commutativity of the generating functions of the classical integrals with respect to the both linear and quadratic Poisson brackets may be shown using theory of compatible Poisson brackets only.

The following theorem holds true:

Theorem 3.2 Let $D(\lambda)$ be a determinant of the gl(n)-valued "monodromy" matrix $\mathcal{T}(\lambda)$ satisfying the brackets (29). Then $D(\lambda)$ is a generating function of the Casimir function of the bracket (29) for all types of the nondegenerated $r$-matrices $r(\lambda-$ $\mu) \in \operatorname{sl}(n) \otimes \operatorname{sl}(n)$.

Proof. For the proof of the Theorem we will need the following Lemma that provides a classical $K(\lambda) \neq 1$ analogue of the known formula [18] that connect the "twisted classical Reflection Equation Algebra" with the corresponding "classical" limit of quantum group:

Lemma 3.1 Let matrix $T(\lambda)$ satisfy the Poisson brackets (18) and constant matrix $K(\lambda)$ satisfy condition (31). Then:

(1) If $\sigma(X)=X$ then the matrix

$$
\mathcal{T}(\lambda)=T(\lambda) K(\lambda) T^{-1}(-\lambda)
$$

satisfy the Poisson brackets (29).

(2) If $\sigma(X)=-X^{t}$, where superscript " $t$ " denotes the anti-automorphism of $g l(n)$ as associative algebra, then the matrix

$$
\mathcal{T}(\lambda)=T(\lambda) K(\lambda) T^{t}(-\lambda)
$$

satisfy the Poisson brackets (29). 
Proof. In the case (1), using classical Yang-Baxter equations (18), it is easy to derive the following equalities:

$$
\begin{gathered}
\left\{T_{1}^{-1}(-\lambda), T_{2}^{-1}(-\mu)\right\}_{2}=-\left[r_{12}(\mu-\lambda), T_{1}^{-1}(-\lambda) T_{2}^{-1}(-\mu)\right], \\
\left\{T_{1}(\lambda), T_{2}^{-1}(-\mu)\right\}_{2}=T_{1}(\lambda) r_{12}(\lambda+\mu) T_{2}^{-1}(-\mu)-T_{2}^{-1}(-\mu) r_{12}(\lambda+\mu) T_{1}(\lambda), \\
\left\{T_{1}^{-1}(-\lambda), T_{2}(\mu)\right\}_{2}=-T_{1}^{-1}(-\lambda) r_{12}(-\lambda-\mu) T_{2}(\mu)+T_{2}(\mu) r_{12}(-\lambda-\mu) T_{1}^{-1}(-\lambda) .
\end{gathered}
$$

In the case (2), in the analogous way, using classical Yang-Baxter equations (18) and the property of $\sigma$ to be minus anti-automorphism of $g l(n)$ it is straightforward to obtain that:

$$
\begin{gathered}
\left\{T_{1}^{t_{1}}(-\lambda), T_{2}^{t_{2}}(-\mu)\right\}_{2}=-\left[r_{12}^{\sigma_{1} \sigma_{2}}(\mu-\lambda), T_{1}^{t_{1}}(-\lambda) T_{2}^{t_{2}}(-\mu)\right], \quad \text { (45a) } \\
\left\{T_{1}(\lambda), T_{2}^{t_{2}}(-\mu)\right\}_{2}=T_{1}(\lambda) r_{12}^{\sigma_{2}}(\lambda+\mu) T_{2}^{t_{2}}(-\mu)-T_{2}^{t_{2}}(-\mu) r_{12}^{\sigma_{2}}(\lambda+\mu) T_{1}(\lambda), \quad \text { (45b) } \\
\left\{T_{1}^{t_{1}}(-\lambda), T_{2}(\mu)\right\}_{2}=-T_{1}^{t_{1}}(-\lambda) r_{12}^{\sigma_{1}}(-\lambda-\mu) T_{2}(\mu)+T_{2}(\mu) r_{12}^{\sigma_{1}}(-\lambda-\mu) T_{1}^{t_{1}}(-\lambda) .
\end{gathered}
$$

Now the Lemma is proved by direct calculation, using the Leibnitz rule for the Poisson bracket, relations (18), (44), (45) and (31).

In order to prove the theorem it is necessary to show that $\{D(\lambda), \mathcal{T}(\mu)\}_{2}=0$. By virtue of the multiplicative properties of the determinant and its invariance with respect to the antiautomorphism $t$ we obtain that $D(\lambda)=k(\lambda) d(\lambda) d^{-1}(-\lambda)$ (if $\sigma=1$ ) or $D(\lambda)=k(\lambda) d(\lambda) d(-\lambda)$ (if $\sigma$ is minus antiautomorphism of $g l(n)$ ). On the other hand, using the fact that $d(\lambda)$ is a generating function of the Casimir functions of the bracket (18) we obtain:

$$
\begin{aligned}
& \{d( \pm \lambda), T(\mu)\}_{2}=\left\{d( \pm \lambda), T^{-1}(-\mu)\right\}_{2}= \\
& \quad=\left\{d^{-1}(-\lambda), T(\mu)\right\}_{2}=\left\{d^{-1}(-\lambda), T^{-1}(-\mu)\right\}_{2}=0 .
\end{aligned}
$$

Keeping in mind that $K(\lambda)$ is a constant (with respect to the Poisson bracket) matrix and making use of the Lemma 3.1 we obtain that $\{D(\lambda), \mathcal{T}(\mu)\}_{2}=0$.

Theorem is proved.

Remark 14. Due to the Remark 11 the Lemma 3.1 may be viewed as a proof (an alternative to the direct calculational one) of the fact that formula (29) correctly defines Poisson bracket. 
From the Theorems 3.2 and 2.1 follows the next statement:

Proposition 3.2 Denote by $D_{k}(\mathcal{T}(\lambda), K(\lambda))$ the coefficients of the decomposition: $\operatorname{Det}(\mathcal{T}(\lambda)+\eta K(\lambda))=k(\lambda) \sum_{k=0}^{n} D_{k}(\mathcal{T}(\lambda), K(\lambda)) \eta^{k}$, where $k(\lambda) \equiv \operatorname{det} K(\lambda)$. Then $D_{k}(\mathcal{T}(\lambda), K(\lambda))$ are generators of an Abelian subalgebra with respect to the linear and quadratic r-matrix brackets (33) and (29), i.e.:

$$
\left\{D_{k}(\mathcal{T}(\lambda), K(\lambda)), D_{l}(\mathcal{T}(\mu), K(\mu))\right\}_{2}=\left\{D_{k}(\mathcal{T}(\lambda), K(\lambda)), D_{l}(\mathcal{T}(\mu), K(\mu))\right\}_{1}=0
$$

Remark 15. The set of functions $\left\{D_{k}(\mathcal{T}(\lambda), K(\lambda))\right\}$ defines the same algebra of integrals as the set of functions $\left\{\operatorname{tr}\left(\mathcal{T}(\lambda) K^{-1}(\lambda)\right)^{k}\right\}$ and they are connected by the well-known Newton formulas. The commutativity of functions from the last set may be also proved directly using the condition (31), explicit form of brackets (29) and brackets (33).

Remark 16. Note, that we have constructed different sets of functions $\left\{D_{k}(\mathcal{T}(\lambda)\right.$, $K(\lambda))$ \} corresponding to different elements $K(\lambda)$ commuting with respect to the same quadratic brackets (29)! Commutativity of each of these sets of functions is closely connected with the existence of the corresponding linearization of the bracket (29) in the neighborhood of the point $K(\lambda)$.

In order to identify the hamiltonian flows with respect to the different brackets we need to decompose the generating functions $D_{k}(\mathcal{T}(\lambda), K(\lambda))$ with respect to the spectral parameter $\lambda$. Using the fact that $\mathcal{T}(\lambda)$ is meromorphic functions this can always be done using the decomposition in Laurent power series. In the special cases [15] one can also use the other decompositions. Nevertheless, we will not do these decompositions explicitly, leaving all the expressions in the "convoluted" form of the generating functions.

Using the Proposition 2.3 we obtain the following Proposition:

Proposition 3.3 Hamiltonian equations of motion with respect to the bracket (29) with a hamiltonian being one of the functions obtained by a decomposition of the function $D_{k}(\mathcal{T}(\lambda), K(\lambda)), k<n$, coincide with the hamiltonian equations of motion with respect to the brackets (33) with the hamiltonian being the corresponding function obtained by the decomposition of the function $-D_{k-1}(\mathcal{T}(\lambda), K(\lambda))$, i.e. on the level of generating functions the following equality is true:

$$
\left\{D_{k}(\mathcal{T}(\lambda), K(\lambda)), \mathcal{T}(\mu)\right\}_{2}=-\left\{D_{k-1}(\mathcal{T}(\lambda), K(\lambda)), \mathcal{T}(\mu)\right\}_{1}
$$

In the case $k=n-1$ this Proposition implies the following Corollary:

Corollary 3.3 Hamiltonian equations of motion with respect to the bracket (29) with a hamiltonian being one of the functions obtained by a decomposition of the function $\operatorname{tr}\left(\mathcal{T}(\lambda) K^{-1}(\lambda)\right.$ coincide with the hamiltonian equations of motion with respect to the brackets (33) with the hamiltonian being the corresponding function 
obtained by the decomposition of the function $\frac{1}{2} \operatorname{tr}\left(\mathcal{T}(\lambda) K^{-1}(\lambda)\right)^{2}$, i.e. on the level of generating functions the following equality is true:

$$
\left\{\operatorname{tr}\left(\mathcal{T}(\lambda) K^{-1}(\lambda)\right), \mathcal{T}(\mu)\right\}_{2}=\frac{1}{2}\left\{\operatorname{tr}\left(\mathcal{T}(\lambda) K^{-1}(\lambda)\right)^{2}, \mathcal{T}(\mu)\right\}_{1}
$$

Proof. In order to derive this statement it is sufficient to put in the previous proposition $k=n-1$, use Newton identities and the fact that function $\operatorname{tr}\left(\mathcal{T}(\lambda) K^{-1}(\lambda)\right)$ is a Casimir function of the linear $r$-matrix bracket (33).

\section{Conclusion and Discussion}

In the present paper we have shown that for a general quadratic Poisson bracket it is possible to define many associated linear Poisson brackets - its linearizations in the neighborhood of special points. We prove that the constructed linear Poisson brackets are always compatible with the initial quadratic Poisson bracket. Using the famous Lenard-Magri scheme we obtain mutually commuting with respect to the both brackets "integrals" starting from Casimir functions of the initial quadratic brackets. We show, that the hamiltonian dynamics with respect to one of these hamiltonians of degree $k$ and quadratic bracket can be re-written in terms of hamiltonian dynamics with respect to the corresponding linear bracket and the other one of these hamiltonians of the degree $k+1$.

We apply the obtained results to the cases of the standard quadratic $r$-matrix bracket and classical "twisted reflection algebra" bracket. We show that in the last case there are a lot of non-equivalent linearizations of the classical twisted reflection algebra bracket and all of them are compatible with initial quadratic bracket. In the both cases we show that generating functions of the classical integrals may be obtained using the decomposition of the "shifted" Casimir function (determinant of the monodromy matrix) of the corresponding quadratic Poisson bracket. In the first case this fact may be viewed as kind of classical explanation of the trick with the "quantum argument shift" of [27] (if the classical $r$-matrix is rational) and one more "classical" argument for the support of the hypothesis of [30] in the case of general classical $r$-matrix. In the second case it may give a hint how to quantize "higher Gaudin hamiltonians" associated with the non-skew-symmetric $r$-matrix $r^{\sigma, K}(\lambda, \mu)$ (see [23]).

Acknowledgements The research described in this paper was partially supported by the FrenchUkrainian project "Dnipro" and the Italian-Russian project "Einstein". The first author is grateful to SISSA, where the final version of this paper was prepared, and the second author is grateful to the University of Angers, where the idea of this paper was conceived, for the warm hospitality.

V.R. much acknowledges the invitation to the Abel Symposium 2008 at Tromso and he is thankful to the Organizing Committee for their excellent job and for very fruitful time during the Symposium. 


\section{References}

1. F. Magri Journal of Math. Physics, 19, 1156-1162 (1978).

2. A. Bolsinov Izv. Akad. Nauk SSSR Ser. Mat. 55, 68-92(1991).

3. I.M. Gelfand, I. Zakharevich J. of Func. Anal 99, 150-157, (1991).

4. I.M. Gelfand, I. Zakharevich Selecta Math. 6, no 2, 131-183, (2000).

5. Y. Kosmann-Schwarzbach, F. Magri Ann. Inst. H. Poincare, 53, No 1, 35 (1990).

6. Y. Kosmann-Schwarzbach, F. Magri Journal of Math. Physics, 37, 6137-6197 (1996).

7. I. Golubchik, V. Sokolov Funct Ann and Appl, 36, no 3, 172 (2002).

8. D. Gurevich, V. Rubtsov Teoret. Mat. Fiz. 103, no 3, 476-488 (1995).

9. D. Gurevich, P. Pyatov, P. Saponov Algebra i Analiz 20, no 2, 70-133 (2008).

10. E. Sklyanin preprint LOMI E-3-79 (1979).

11. M. Semenov Tian-Shansky Funct An and Apl, v 17, 259 (1983).

12. E. Sklyanin, P. Kulish Zapiski LOMI, 95, 129 (1980).

13. A.A. Belavin, V. Drienfield Funct. annalysis and its appl., 16, no 3, 1-29 (1982).

14. B. Khesin, A. Levin, M. Olshanetsky Comm. Math. Phys., 250, 581-612, (2004).

15. A. Levin, M. Olshanetsky, A. Zotov Commun. Math. Phys. 268 67-103 (2006).

16. Yu. Chernyakov, A. Levin, M. Olshanetsky, A. Zotov "Quadratic algebras related to elliptic curves" nlin. SI/07101072.

17. A.A. Belavin Funct. annalysis and its appl., 14, no 4, p. 18-26, (1980).

18. E. Sklyanin Journal of Physics A. 21, 2375 (1988).

19. L. Mezincesku, R. Nepomechie Journal of Physics A. 25, 2553 (1992).

20. J.M. Maillet Phys Lett B, 167, 401 (1986).

21. O. Babelon, C.M. Viallet Phys. Lett B. 237, 411 (1990).

22. J. Avan, M. Talon Phys. Lett. B,241 77 (1990).

23. T. Skrypnyk Journal of Mathematical Physics 48, no 12, 113521 (2007).

24. A. Odesskij, V. Sokolov Journal of Mathematical Physics 47, no 1, (2006).

25. E. Sklyanin Funct. an. i ego pril. 16, no 4, 27 (1982)

26. A. Stolin Comm. Math. Phys. 141, 533 (1991)

27. D. Talalaev "Quantization of the Gaudin system", hep-th/0404153.

28. A. Molev, M. Nazarov, G. Olshansky Russ. Math. Surveys 51, 205 (1996)

29. A. Hurwitz, R. Courant Theory of functions, Springer, Berlin, 1964, 648 p.

30. A. Chervov, D. Talalaev "Quantum spectral curves, quantum integrable systems and the geometric Langlands correspondence" hep-th/0604128 